\title{
Kinder- und jugendpsychiatrische stationsäquivalente Behandlung (StäB): Therapieoption - für alle oder für wenige?
}

\author{
Isabel Boege ${ }^{1,2}$, Renate Schepker ${ }^{1}$, Dieter Grupp ${ }^{1}$ und Jörg M. Fegert ${ }^{2}$ \\ ZfP Südwürttemberg, Akademisches Lehrkrankenhaus der Universität Ulm, Ravensburg, Deutschland \\ KJPP Universität UIm, Ulm, Deutschland
}

\begin{abstract}
Zusammenfassung: Fragestellung: Aufsuchende stationsäquivalente Behandlung (StäB) ist seit dem 01.01.2017 in Deutschland möglich und seit dem 01.01.2018 abrechenbar. Dennoch wird StäB unter Infragestellung der Machbarkeit der Rahmenbedingungen derzeit nur an wenigen Standorten der Kinder- und Jugendpsychiatrie und Psychotherapie in Deutschland regelhaft angeboten. Ziel war es, anhand der ersten 58 stationsäquivalent behandelten Kinder und Jugendlichen am ZfP Südwürttemberg (2018-2019) die Machbarkeit und Kosten von StäB zu evaluieren. Methodik: Es wurden alle seit dem 01.01.2018 konsekutiv stationsäquivalent behandelten Fälle eingeschlossen und anhand von rein deskriptiven Analysen in SPSS.25 evaluiert. Ergebnisse: Die durchschnittliche Behandlungsdauer betrug 37.95 Tage (SD 15.35), 86.2\% aller Familien beendeten die Behandlung regelgerecht, es kam nur bei drei Patientinnen und Patienten zu einem einvernehmlichen Behandlungsabbruch, fünf mussten in eine stationäre Behandlung verlegt werden. Es wurden primär internalisierende Störungen indiziert (70.7\%), während externalisierende Störungen nur ein Viertel der Behandlungsfälle ausmachten (27.8\%). Kosten einer StäB beliefen sich im Durchschnitt auf $8779,25 €$. Schlussfolgerungen: StäB stellt eine neue Behandlungsform im familiären Umfeld dar, welche täglich stattfindet. Multiprofessionalität der Behandlung ist Voraussetzung. Eine Umsetzung in den gegebenen Rahmenbedingungen ist möglich. Eine Akzeptanz von den Familien ist gegeben, die durchschnittliche Behandlungsdauer liegt leicht über dem stationären Bundesdurchschnitt.
\end{abstract}

Schlüsselwörter: Hometreatment, Stationsäquivalente Behandlung, Kinder und Jugendliche; Aufsuchende Behandlung, Versorgung

Intensive outpatient treatment - a therapy option for all patients in child and adolescent psychiatry or just for a few?

Abstract: Objective: "Child and adolescent psychiatric ward-equivalent treatment" (= stationsäquivalente Behandlung, StäB) is an intensive daily home treatment that has now become available in Germany. Conditions for StäB services were negotiated were negotiated, authorized and came into force by German healthcare commissioners and hospitals on 1 January 2018. However, to date few hospitals in Germany offer StäB, and its feasibility has been questioned. This study evaluates the first 58 cases of StäB in the Department of Child and Adolescent Psychiatry, ZfP Südwürttemberg (2018-2019). Method: All patients admitted consecutively to StäB from 1 January 2018 to 15 August 2019 were included and evaluated solely using descriptive analyses in SPSS.25. Results: The average length of stay in StäB was 37.95 days (SD 15.35). $86.2 \%$ of the families completed treatment, with 3 patients discontinuing treatment and 5 patients needing inpatient admission. Unlike in the literature, internalising disorders predominated $(70.7 \%)$, whereas externalizing disorders were diagnosed in only $27.8 \%$ of the patients. The cost of treatment in StäB was on average $8779.25 €$. Conclusions: StäB is a viable and well-accepted new treatment alternative for patients requiring inpatient admission. The multiprofessionality of treatment as well as daily contacts are required. Feasibility under the conditions set is given. The average length of stay is slightly longer, but the costs are lower than those of inpatient treatment.

Keywords: home treatment, children and adolescents, child and adolescent psychiatry, outreach teams, mental health problems

\section{Ausgangslage}

Psychische Störungen gehören zu den häufigsten Erkrankungen bei Kindern und Jugendlichen. Prävalenzzahlen rangieren weltweit zwischen 9 und 22\% (Bark- mann \&Schulte-Markwort, 2010; Belfer, 2008; Hölling et al., 2014; Merikangas et al., 2010; Ravens-Sieberer, Wille \& Erhard, 2008).

In der KiGGS-Studie (erste Erhebung von 2003 bis 2006, zweite Erhebung von 2009 bis 2012), welche re- 
präsentativ für Deutschland den Gesundheitszustand deutscher Kinder und Jugendlicher erhob, fanden sich bei ca. jedem fünften Kind/Jugendlichen psychische Auffälligkeiten (Welle 1: 20.0\%, Welle 2: 20.2\%; Hölling et al., 2014; Hölling, Erhard, Ravens-Sieberer \& Schlack, 2007). Unabhängig von der gestellten Diagnose befanden sich sowohl zum Zeitpunkt der ersten Erhebung als auch zum Zeitpunkt der zweiten Erhebung weniger als die Hälfte der Kinder in einer Behandlung (Hölling et al., 2014), obwohl psychische Störungen im Kindes- und Jugendalter oftmals mit erheblichen Beeinträchtigungen der sozialen, schulischen und beruflichen Integration des Kindes/Jugendlichen einhergehen. Belastungen des familiären, schulischen oder erweiterten sozialen Umfelds können daraus resultieren (Mattejat et al., 2003; Wille, Bettge, Wittchen \& Ravens-Sieberer, 2008). Eine frühe effiziente Behandlung ist deswegen sowohl zur Entlastung des individuellen Leidensdruckes als auch hinsichtlich der Gefahr der Chronifizierung, mit langfristig entstehenden Kosten im Gesundheitssystem, von hoher Relevanz.

Nach einer früheren Studie kann es von den ersten Symptomen bis zur ersten angemessenen Behandlung zu einer Behandlungsverzögerung von bis zu 6;8 Jahren kommen. Die Verzögerung des Behandlungsbeginns wurde dabei als signifikant invers mit einem frühen Ersterkrankungsalter assoziiert aufgezeigt (Mattejat et al., 2003). Als ursächlich für eine fehlende oder späte Hilfesuche aufseiten der Patientenfamilien werden u.a. Angst vor Stigmatisierung, Unkenntnis von möglichen Hilfen oder strukturelle Schwierigkeiten angegeben (Ravens-Sieberer, Schulte-Markwort, Bettge \& Barkmann, 2002). Auf der anderen Seite hält das Versorgungssystem nicht immer passende, zeitnahe, effektive und effiziente Versorgungsangebote vor. Etwas mehr als die Hälfe der niedergelassenen Kinder- und Jugendpsychiaterinnen und -psychiater bieten multiprofessionelle Behandlung über die Sozialpsychiatrievereinbarung an, im Rahmen derer unter ärztlicher Leitung auch nichtärztliche Leistungen (psychologische, psychosoziale, heilpädagogische Unterstützung) in einer Praxis angeboten werden können. Diese Arztgruppe aber ist für $20 \%$ der Patientinnen und Patienten mit (PKW-)Fahrzeiten von über 1 Stunde am schlechtesten erreichbar (Gemeinsamer Bundesausschuss, 2018). Eine stationäre Aufnahme ist nach eingetretener Chronifizierung ohne Behandlung oftmals nicht die gewünschte Lösung, eine ambulante Behandlung wiederum gegebenenfalls nicht mehr ausreichend. Hier kann Hometreatment eine Alternative zu einer stationären Behandlung darstellen.

Im Folgenden werden nur diese Fälle - Kinder und Jugendliche mit stationärer Behandlungsindikation - betrachtet.

\section{Versorgungsangebote der krankenhausbasierten Kinder- und Jugendpsychiatrie und Psychotherapie in Deutschland}

An 147 Krankenhäusern bieten Kliniken bzw. Abteilungen für Kinder- und Jugendpsychiatrie und Psychotherapie (KJPP) eine hochintensive stationäre und teilstationäre Versorgung an. Mit den vorgehaltenen stationären kinder- und jugendpsychiatrischen Behandlungsplätzen steht Deutschland dabei europaweit an der Spitze (Signorini et al., 2017). 61091 Kinder und Jugendliche wurden 2017 stationär aufgenommen und mit einer durchschnittlichen Verweildauer von 34.4 Tagen stationär behandelt (Statistisches Bundesamt, 2018).

Parallel kam es in den letzten 13 Jahren zu einem deutlichen Zuwachs an tagesklinischen Plätzen (2004: 1454 Plätze vs. 2017: 3652 Plätze $=+151 \%$ ). Teilstationäre Angebote müssen dabei für die Patientinnen und Patienten in einem realistischen Zeitraum erreichbar sein. Der Ausbau an tagesklinischen Plätzen führte allerdings nicht im erwarteten Ausmaß zur Entlastung vollstationärer Kapazitäten, stattdessen scheinen durch die teilstationären Plätze offenbar insgesamt mehr Kinder und Jugendliche mit psychischen Auffälligkeiten erreicht zu werden (Statistisches Bundesamt, 2018; Warnke \& Lehmkuhl, 2011). Die regionale Verteilung der Kapazitäten, rechnet man sie auf Einwohnerinnen und Einwohner unter 18 Jahre, ist dabei höchst unterschiedlich.

Ambulant hingegen werden in aller Regel an den Krankenhäusern nur niederfrequente diagnostische bzw. vorund nachstationäre Behandlungstermine in den Institutsambulanzen vorgehalten. Dabei müssen für die Indikation einer Behandlung an einer psychiatrischen Institutsambulanz Schwere, Dauer und/oder die Notwendigkeit einer krankenhausnahen Behandlung gegeben sein.

In Deutschland liegt für Hometreatment, als Alternative zu einer stationären Behandlung, inzwischen eine hinreichend gute Evidenz vor (Boege, Corpus \& Schepker, 2014; Boege, Corpus, Schepker, Kilian \& Fegert, 2015; Corpus, Schepker, Fegert \& Boege, 2014; Mattejat, Hirt, Wilken, Schmidt \& Remschmidt, 2001; Remschmidt, Schmidt, Mattejat, Eisert \& Eisert, 1988; Schmidt, Lay, Göpel, Naab \& Blanz, 2006). Hometreatment konnte bisher aber nicht als Regelleistung angeboten werden, da es keine Gesetzesgrundlage für eine Finanzierung der aufsuchenden Behandlungsform durch die Kassen gab.

Erst jetzt mit dem „Gesetz zur Weiterentwicklung der Versorgung und der Vergütung für psychiatrische und psychosomatische Leistungen (PsychVVG)“, welches am 01.01.2017 in Kraft trat, wurde die Grundlage für alle Versichertengruppen geschaffen, alternativ zur stationären Behandlung intensives Hometreatment in Form von stati- 
onsäquivalenter Behandlung (StäB) als Krankenhausbehandlung anzubieten. Die Bundesrahmenvereinbarung zwischen dem GKV-Spitzenverband, dem Verband der Privaten Krankenversicherung und der Deutschen Krankenhausgesellschaft schuf die Basis für die regionalen Kassenverhandlungen und trat am 01.01.2018 in Kraft (Deutsche Krankenhausgesellschaft, 2017).

Bisher wird StäB aber noch nicht regelhaft in allen Regionen Deutschlands angeboten. Es fehlen etablierte Routinen für aufsuchende Tätigkeiten. Strukturelle Fragen der Organisation eines StäB-Teams, aber auch Fragen nach Annahme durch die Familien und Wirksamkeit von Interventionen werden gestellt. Ziel dieser Arbeit ist es deswegen, anhand der ersten 58 Patientinnen und Patienten, die vom „StäB-Team-KiJu“ des ZfP Südwürttemberg kontinuierlich aufgenommen und in den Jahren 2018 bis 2019 behandelt wurden, die Machbarkeit und Finanzierbarkeit von StäB zu evaluieren.

\section{Rahmenbedingungen einer StäB am ZfP Südwürttemberg}

In der Abteilung für Psychiatrie und Psychotherapie des Kindes- und Jugendalters, ZfP Südwürttemberg, wurden seit dem 01.01.2018 neben der stationären (30 Plätze) und teilstationären Regelversorgung (10 Plätze) zunächst vier bettenäquivalente Plätze (ab dem 01.01.2018) und ab dem 01.01.2019 sechs bettenäquivalente Plätze für StäB vorgehalten. Patientinnen und Patienten kamen für eine StäB infrage, wenn eine Indikation für eine stationäre kinderund jugendpsychiatrische Behandlung vorlag und sie in einem PKW-Umkreis von 30 Minuten (minutenscharf nach Routenplaner) vom Hauptstandort des ZfP Südwürttemberg wohnten.

Das StäB-Team-KiJu am ZfP Südwürttemberg besteht aus einer Fachärztin für KJPP (10\% Stellenanteil), einer Assistenzärztin (50\%), einer Psychologin (70\%), fünf Mitarbeitern des Pflege- und Erziehungsdienstes (PED; mit insgesamt $280 \%$ Stellenanteil) einer Organisationsfachwirtin $(40 \%)$ und drei Fachtherapeuten (Heilpädagogik, Kunsttherapie und Ergotherapie mit insgesamt 120\% Stellenanteil; Stand 01.01.2019, für sechs Patienten). Ein individueller Schwerpunkt des Teams liegt auf erlebnispädagogischen Angeboten. Geleistet wird im häuslichen Kontext das gesamte Spektrum einer stationären kinder- und jugendpsychiatrischen Behandlung.

Die einzelnen StäB-Kontakte können bestehen aus: Einzelgesprächen, Familiengesprächen, gezielter Anleitung von Bezugspersonen in dem Herkunftsmilieu, Entspannungsverfahren, Psychopharmakotherapie, (störungsspezifischer) Psychoedukation, Helferkonferenzen (z.B. Jugendhilfe, Schule, Arbeitsamt), Anleitung von Bezugsper- sonen im Umgang mit der Patientin oder dem Patienten, alltagsbezogenes Training, Anleitung und Förderung der Selbstständigkeit, gelenkte Freizeitaktivitäten, Medienpädagogik, Erlebnispädagogik/-therapie, Ergotherapie, Kreativtherapien, Bewegungstherapie. Gruppentherapien sind möglich. Die Schule für Kranke kann von StäB-Patientinnen und -Patienten genutzt werden, eine entsprechende Erweiterung der Schulkapazität fand statt.

Die stationsäquivalenten Plätze waren in den letzten anderthalb Jahren kontinuierlich belegt, es besteht eine Warteliste.

\section{Ablauf einer StäB}

Eine StäB wird zunächst von Kolleginnen und Kollegen der Institutsambulanz oder der Station (z. B. bei Krisenpatientinnen und -patienten) oder von niedergelassenen Kolleginnen und Kollegen indiziert. Auch können sich Eltern direkt an das StäB-Team wenden.

Bei Interesse an StäB erfolgt dann bei einem Mitarbeiter aus dem StäB-Team ein Erstgespräch in der Ambulanz, in dem das Konzept StäB der Familie vorgestellt wird und die Indikation und auch Motivation des Kindes bzw. Jugendlichen sowie der Eltern überprüft werden. Alle im Haushalt lebenden Personen, die $\geq 18$ Jahre alt sind, müssen dabei mit der StäB des Kindes oder Jugendlichen einverstanden sein. Eine StäB dauert in der Regel 6 Wochen und findet täglich statt, auch an Wochenenden und Feiertagen. Alle beteiligten Berufsgruppen leisten in diesem Rahmen Wochenenddienste. Im Anschluss an eine StäB werden den Familien noch 1-mal/Woche bis maximal 2-mal/Woche aufsuchende Nachbetreuungstermine von den ihnen bekannten StäB-Mitarbeiterinnen und -Mitarbeitern für maximal 6 Monate angeboten.

Entscheiden sich das Kind bzw. der Jugendliche sowie die Eltern für eine StäB, erfolgt, sobald ein Platz frei wird, ein Aufnahmegespräch in der Familie durch den für die Fallführung vorgesehenen Kollegen gemeinsam mit der Fachärztin für KJPP. Die Fallführung kann dabei von der Assistenzärztin, der Psychologin oder einem der Mitarbeiter aus dem Pflege- und Erziehungsdienst übernommen werden. Fallführung beinhaltet, dass die jeweilige Person für die Behandlungsplanung zuständig ist, die praktische Koordination der StäB-Maßnahmen für die Familie übernimmt und erste Ansprechpartnerin bei Fragen ist. Das Aufnahmegespräch in der Familie dient dazu, die Eignung des Wohnumfelds für Einzel- und Familiengespräche zu überprüfen, die Behandlungsziele fachärztlich festzulegen sowie der Familie den ersten, anhand der Vorinformationen erstellten, Wochenplan vorzustellen, auf dem für jeden Tag die Art des Kontaktes (Einzelgespräch, Familiengespräch, Fachtherapie, Erlebnisthera- 
pie) sowie der Name der durchführenden Person vermerkt ist. Die Familie erhält zudem eine Liste mit den Namen und den Berufsgruppen der jeweiligen StäB-Mitarbeiter und -Mitarbeiterinnen. In der Regel finden alle Maßnahmen aufsuchend statt, Ausnahmen sind erlebnistherapeutische Maßnahmen (z.B. Bogenschießen, Hochseilgarten) sowie eine wöchentliche Gruppentherapie zu Schulung sozialer Kompetenzen. Diese finden im ZfP Südwürttemberg statt.

In den wöchentlichen multiprofessionellen Fallbesprechungen werden regelmäßig die neuen Wochenpläne erstellt, die Behandlungsziele überprüft sowie supervisorische bzw. methodische Fragen fachärztlich geklärt. Die Erreichbarkeit mindestens eines Mitglieds des Behandlungsteams ist werktags im Rahmen des üblichen Tagesdienstes (8:00-18:00 Uhr) über ein Diensthandy sichergestellt. Darüber hinaus ist jederzeit (24 Stunden an 7 Tagen in der Woche) eine bestehende ärztliche Eingriffsmöglichkeit durch den Dienstarzt der Abteilung gewährleistet. Bei kurzfristiger Zustandsverschlechterung kann so umgehend mit einer vollstationären Aufnahme reagiert werden.

Jedes Teammitglied ist mit Dienstsmartphone und Dienstlaptop ausgestattet, um eine taggleiche dezentrale Dokumentation in der elektronischen Patientenakte und
Informationsweitergabe zu gewährleisten; alternativ gibt es ausreichend Dokumentationsplätze in den jeweiligen Büros. Die OPS (Operationen- und Prozedurenschlüssel) -Kodes sind für jeden Tag berufsgruppenspezifisch und minutengenau anzugeben. Mobilität wird durch eine entsprechende Anzahl an Fahrzeugen gewährleistet.

Damit entspricht die StäB am ZfP Südwürttemberg den Bedingungen der Bundesrahmenvereinbarung zwischen dem GKV-Spitzenverband, dem Verband der Privaten Krankenversicherung und der Deutschen Krankenhausgesellschaft (2017; vgl. Tabelle 1).

\section{Methodik}

Alle Patientinnen und Patienten, die konsekutiv zwischen dem 01.01.2018 und dem 15.08.2019 stationsäquivalent behandelt wurden und deren Behandlung inzwischen abgeschlossen ist, wurden in die Auswertung eingeschlossen. Die Daten wurden retrospektiv anonymisiert erfasst. Ein Ethikvotum wurde eingeholt und am 21.08.2019 unter der Nummer 223/19 von der Ethikkommission der Universität Ulm bewilligt.

Tabelle 1. Kriterien einer stationsäquivalenten Behandlung (StäB).

\begin{tabular}{|c|c|}
\hline & Kriterien einer StäB \\
\hline 1 & $\begin{array}{l}\text { Therapiezielorientierte Behandlung durch ein mobiles multiprofessionelles Team unter Leitung einer Fachärztin oder eines Facharz- } \\
\text { tes für Kinder- und Jugendpsychiatrie und Psychotherapie. }\end{array}$ \\
\hline 2 & Drei Berufsgruppen werden vorgehalten (im Team oder im Krankenhaus). \\
\hline 3 & Ein Einverständnis aller mit im Haushalt lebenden > 18-jährigen Mitbewohnerinnen und Mitbewohner muss vorliegen. \\
\hline 4 & $\begin{array}{l}\text { Durchführung einer wöchentlichen ärztlichen Visite (bei StäB an mehr als } 6 \text { Tagen in Folge) im direkten Patientenkontakt, in der Regel } \\
\text { im häuslichen Umfeld. Der Facharztstandard ist zu gewährleisten. }\end{array}$ \\
\hline 5 & $\begin{array}{l}\text { Durchführung einer wöchentlichen multiprofessionellen Fallbesprechung zur Beratung des weiteren Behandlungsverlaufs (bei StäB } \\
\text { an mehr als } 6 \text { Tagen in Folge), in die mindestens drei der an der Behandlung beteiligten Berufsgruppen ggf. unter Einbeziehung koope- } \\
\text { rierender Leistungserbringer nach } \$ 115 \mathrm{~d} \text { Abs. } 1 \text { Satz } 3 \text { SGB (Sozialgesetzbuch) V einbezogen werden. Die Fallbesprechung kann un- } \\
\text { ter Zuhilfenahme von Telekommunikation geschehen. }\end{array}$ \\
\hline 6 & $\begin{array}{l}\text { Behandlung auf der Grundlage eines individuellen Therapieplans, orientiert an den Möglichkeiten und dem Bedarf der Patientin bzw. } \\
\text { des Patienten. }\end{array}$ \\
\hline 7 & $\begin{array}{l}\text { Es erfolgt mindestens ein direkter Patientenkontakt durch mindestens ein Mitglied des multiprofessionellen Teams pro Tag. Kommt } \\
\text { ein direkter Kontakt nicht zustande aus Gründen, die die Patientin oder der Patient zu verantworten hat, zählt der unternommene } \\
\text { Kontaktversuch dennoch als direkter Patientenkontakt. }\end{array}$ \\
\hline 8 & $\begin{array}{l}\text { Die Erreichbarkeit mindestens eines Mitglieds des Behandlungsteams ist werktags im Rahmen des üblichen Tagesdienstes sicherzu- } \\
\text { stellen (Rufbereitschaft). Darüber hinaus ist jederzeit ( } 24 \text { Stunden an } 7 \text { Tagen in der Woche) eine ärztliche Eingriffsmöglichkeit durch } \\
\text { das Krankenhaus zu gewährleisten. Bei kurzfristiger Zustandsverschlechterung muss umgehend mit einer vollstationären Aufnahme } \\
\text { reagiert werden können. }\end{array}$ \\
\hline 9 & $\begin{array}{l}\text { Die einzelnen StäB-Kontakte können bestehen aus: ärztlichen oder psychologischen Einzelgesprächen, Familiengesprächen, geziel- } \\
\text { ter Anleitung von Bezugspersonen aus dem Herkunftsmilieu, Entspannungsverfahren, Psychopharmakotherapie, (störungsspezifi- } \\
\text { sche) Psychoedukation, Helferkonferenzen (z. B. Gemeindepsychiatrische Verbünde [GPV], Jugendhilfe), Anleitung von Bezugsperso- } \\
\text { nen im Umgang mit der Patientin oder dem Patienten, alltagsbezogenes Training, Anleitung und Förderung der Selbstständigkeit, } \\
\text { gelenkten Freizeitaktivitäten, Medienpädagogik, Erlebnispädagogik/-therapie, Ergotherapie, Kreativtherapien, Bewegungstherapie. }\end{array}$ \\
\hline
\end{tabular}


Anhand unseres elektronischen Datenerfassungssystems wurden in einem Datenblatt folgende Daten erhoben: Alter, Geschlecht, ICD-10-Diagnose Achse 1 (International Statistical Classification of Diseases and Related Health Problems; psychiatrische Diagnose) und Achse 5 (psychosoziale Diagnosen), Anzahl der erhaltenen Behandlungselemente (Einzeltherapie, Familientherapie, Fachtherapie, Erlebnispädagogik, Klinikschule), Anzahl der Kontakte nach Berufsgruppe (Ärztin, Psychologin, Pflege- und Erziehungsdienst), Anzahl an notwendigen stationären Aufnahmen nach Beendigung von StäB, Wahrnehmen des Angebots der aufsuchenden Nachbetreuung, Dauer der Nachbetreuung, Kooperation mit umliegenden Institutionen (Schule, Jugendamt) sowie die durchschnittlichen Kosten einer StäB. Diese wurden errechnet aus dem in den Pflegesatzverhandlungen vereinbarten Basistagessatz, einer 40-Minuten-Pauschale für die Fahrzeit sowie die pro Tag von den Mitarbeiterinnen und Mitarbeitern an der Patientin oder am Patienten erbrachte Zeiteinheiten. Sowohl für die Fahrzeit als auch für die Leistung an der Patientin oder am Patienten wurden angelehnt an den OPS-Katalog mit der Kasse vereinbarte Minutenwerte je Berufsgruppe abgerechnet, wobei eine Ärztin höher als eine Psychologin, diese höher als der Pflege- und Erziehungsdienst, jener wiederum höher als die Fachtherapie vergütet wurde. Wenn zwei Mitarbeiterinnen oder Mitarbeiter gemeinsam eine Patientin oder einen Patienten aufsuchten, wurden beide Berufsgruppen sowohl hinsichtlich der Fahrzeit als auch der Zeit an der Patientin bzw. am Patienten abgerechnet.

In SPSS, Version 25, wurden Mittelwerte, $t$-Tests und deskriptive Statistiken berechnet.

\section{Ergebnisse}

Seit dem 01.01.2019 wurden 58 Kinder und Jugendliche im Alter von 8 bis 17 Jahren in stationsäquivalent behandelt, $41.4 \%(n=24)$ weiblich, $58.6 \%(n=34)$ männlich. Familien, die eine StäB wünschten, waren zu den täglich angebotenen Terminen verlässlich zu Hause und nahmen die Termine wahr. Der Altersdurchschnitt bei Kindern (5-13 Jahre, $n=29$ ) lag bei 11;1 Jahren ( $S D$ 1;59), bei Jugendlichen (14-17 Jahre, $n=29$ ) bei 15;3 Jahren $(S D$ 1;14). Diagnostiziert wurden vor allem internalisierende Störungen (70.7\%), wobei die Diagnosen Angststörungen, soziale Phobien, Zwangsstörungen, Anpassungsstörungen bzw. Posttraumatische Belastungsstörungen mit $31 \%$ am häufigsten vorkamen gefolgt von emotionalen Störungen des Kindesalters (F93, 20.7\%; oft verbunden mit Schulverweigerung) sowie depressiven Störungen (F3, 12.1\%). Essstörungen (F5,
6.9\%) und das Asperger-Syndrom (F84.5, 1.7\%) kamen etwas seltener vor. Bei den externalisierenden Störungen, welche $27.8 \%$ der Diagnosen ausmachten, war die Störung des Sozialverhaltens am häufigsten vertreten (F91.x/92.x, 19.0\%), gefolgt von der hyperkinetischen Störung bzw. der hyperkinetischen Störung des Sozialverhaltens (F90.x, 8.6\%).

Bei den im Jahr 2018 stationär aufgenommenen 545 Regelpatientinnen und -patienten zeigte sich eine ähnliche Verteilung der Störungsbilder. Angststörungen, soziale Phobien, Zwangsstörungen, und Anpassungsstörungen bzw. Posttraumatische Belastungsstörungen wurden gleich häufig wie in StäB diagnostiziert (F4, 31\%), gefolgt von emotionalen Störungen des Kindesalters (F93.0, $11 \%)$ und depressiven Störungen (F3, 11\%). Essstörungen (F5) wurden zu 2\% und das Asperger-Syndrom zu $3 \%$ diagnostiziert. Externalisierende Störungen wurden zu 36\% diagnostiziert. Am häufigsten kamen Störungen des Sozialverhaltens vor (F91.x/F92.x, 20 \%), hyperkinetische Störung/hyperkinetische Störung des Sozialverhaltens (F90.x) kam zu 11\% vor. Seltener diagnostiziert wurden Bindungsstörungen (F94.x, 4\%; siehe Abbildung 1).

Fast die Hälfte der Patientinnen und Patienten wiesen zwei oder mehr F-Diagnosen auf (zwei Diagnosen: 29.3\%, drei Diagnosen: $13.8 \%)$. Nicht suizidales selbstverletzendes Verhalten trat bei $15.5 \%$ der Patientinnen und Patienten als Zweitdiagnose mit auf.

Bei den psychosozialen Diagnosen (Z-Diagnosen, Mehrfachnennungen möglich) standen Kontaktanlässe in Bezug auf die Erziehung im Vordergrund (Z62 = 77.6\%) gefolgt von Kontaktanlässen in Bezug auf die Schule $(\mathrm{Z} 55=56.9 \%)$, auf die soziale Umgebung $(\mathrm{Z} 60=29.3 \%)$ sowie auf den engen Familienkreis ( $\mathrm{Z} 63=37.9 \%$; siehe Abbildung 2).

Die durchschnittliche Behandlungsdauer lag bei 37.95 Tagen (min. 2 Tage, max. 80 Tage, SD 15.35). Kinder waren tendenziell etwas kürzer in StäB (36 Tage, $S D$ 17.0) als Jugendliche (39 Tage, $S D 13.6, p=.338$ ).

Eine stationäre Krisenintervention für ein bis zwei Nächte während der StäB war bei drei Patienten nötig. Zu einer Überleitung in einen stationären Aufenthalt, da der Patient oder die Patientin in StäB nicht tragbar war, kam es in fünf Fällen. Drei Behandlungen wurden im gegenseitigen Einverständnis vorzeitig nach 2 bis 3 Wochen beendet. 50 von 58 Patienten $(86.2 \%)$ beendeten somit die StäB regulär. Von diesen 50 Patientinnen und Patienten nahmen 41 Familien (82\%) das Angebot der aufsuchenden Nachbetreuung wahr, 15 (30\%) nahmen diese für ein Quartal wahr, neun (18\%) für zwei Quartale, sechs (12\%) für > drei Quartale, bei 11 (22\%) Patientinnen und Patienten ist die Nachbetreuung aktuell noch nicht abgeschlossen. 

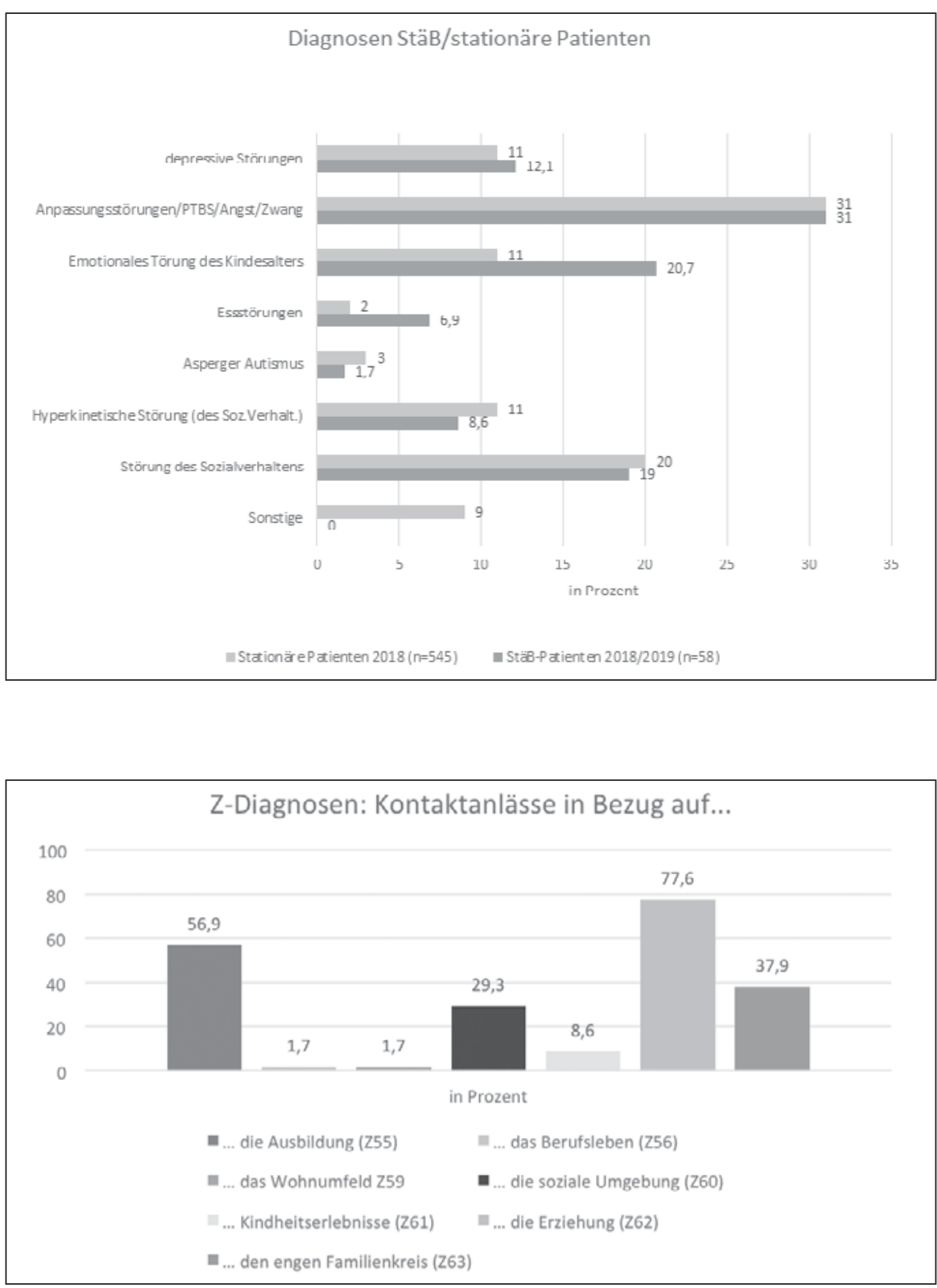

Abbildung 1. Diagnosen StäB-Patienten im Vergleich zu stationären KJPP-Patienten.
Abbildung 2. Psychosoziale Diagnosen bei Kindern und Jugendlichen in StäB.

\section{Angebotene Behandlungselemente}

Es wurden Einzelgespräche, Familiengespräche, Elternanleitung, Fachtherapie (Ergotherapie, Kunsttherapie, Heilpädagogik) sowie Erlebnistherapie indikationsspezifisch angeboten. Durchschnittlich erhielten Patientinnen und Patienten 39.1 Termine, davon 20.9 Einzelgespräche, 6.9 Familiengespräche, 5.5 fachtherapeutische Angebote sowie 5.8 erlebnistherapeutische Einheiten. Jugendliche mit internalisierenden Störungen erhielten signifikant mehr Einzeltherapieangebote als Kinder mit internalisierenden Störungen $(p=.037)$. Dabei wurde der Großteil aller Termine vom Pflege- und Erziehungsdienst angeboten (20.8 Termine), 7.0 Termine erfolgten im Durchschnitt durch den ärztlichen Dienst, 6.4 Termine durch den psychologischen Dienst und 6.9 Termine durch Erlebnispädagogen (Mitarbeiter im PED mit Zusatzausbildung). 22.4\% der Patientinnen und Patienten besuchten zusätzlich die Klinikschule, bei $46.6 \%$ der Patientinnen und Patienten erfolgte eine Kooperation mit der Heimatschule, $31 \%$ benötigten keine weitere Schulintervention. Bei 19\% erfolgte eine Kooperation mit dem Jugendamt (siehe Tabelle 2).

Eine psychopharmakologische Behandlung erfolgte bei $32.6 \%$ der Patientinnen und Patienten. 10.3\% erhielten Antidepressiva (Serotonin-Wiederaufnahmehemmer), 15.5\% erhielten Stimulanzien und 6.8\% Patientinnen und Patienten erhielten Neuroleptika. Die Medikation wurde vom Krankenhaus gestellt. 


\section{Kosten}

Abgerechnet werden konnte eine Basispauschale von $145 € /$ Tag (für das Vorhalten von Räumlichkeiten, EDVTechnik, PKWs und Fahrkosten, Therapiematerial, Kosten der Medikation, von Blut- und EKG-Kontrollen, Rufdienst der Mitarbeitenden) sowie die an der Patientin oder am Patienten vor Ort erbrachte Behandlungsleistung, die pro Berufsgruppe mit 1,02 €/Minute bei Arztleistungen, $0,82 € /$ Minute bei Psychologenleistungen, $0,73 € /$ Minute bei Pflegeleistungen und 0,67 €/Minute bei Fachtherapeutenleistungen bewertet und abgerechnet wurden. Fuhren zwei Berufsgruppen für ein gemeinsames Gespräch in die Familie, wurden beide Berufsgruppen abgerechnet. Für die Fahrzeiten wurde zusätzlich eine Pauschale von 40 min je Minutenwert der Berufsgruppe vereinbart. Fahrtkosten für PKW-Nutzung wurden nicht gesondert erstattet. Bei einer durchschnittlichen Verweildauer von 37.95 Tagen ergab sich so ein durchschnittlicher Gesamtkostenbetrag je Fall von $8779,25 €$, wobei der Berechnungstagessatz im Durchschnitt bei 242,94 € lag (min.: 204,85 €/Tag bis max.: $591,60 € /$ Tag). Eine vollstationäre Behandlung in der Abteilung für Kinder- und Jugendpsychiatrie je nach abgerechneten PEPP- (Pauschalierendes Entgeltsystem Psychiatrie und Psychosomatik) und Basisfallwert lag im Durchschnitt bei $517 € /$ Berechnungstag (min.: $455 €$ ) Tag bis max.: $765 € / \mathrm{Tag}$ ). Jugendliche mit einer externa- lisierenden Störung, welche stationsäquivalent behandelt wurden, waren mit einem durchschnittlichen $\mathrm{Ge}$ samtkostenbetrag von 10232,32 € tendenziell teurer als Kinder mit einer externalisierenden Störung (durchschnittlich 7813,08 €, $p=.142$ ). Dieser Unterschied erreichte aber nur bei den Fahrkosten ein Signifikanzniveau (Kinder 924,80 €, Jugendliche 1370,97 €, $p=.034$ ). Bei Kindern und Jugendlichen mit einer internalisierenden Störung ergab sich dieser Unterschied nicht. In der Gesamtgruppe ergab sich der gleiche tendenzielle Effekt (Gesamtkosten Kinder 8225,58 €, Gesamtkosten Jugendliche 9329,92 $€, p=.246$; Fahrkosten Kinder 895,25 €, Fahrkosten Jugendliche 1143,12 $€, p=.031$ ). Mit der lokal verhandelten Vergütung konnten die Leistungen kostendeckend erbracht werden (siehe Tabelle 3).

\section{Diskussion}

Seit fast einem halben Jahrhundert stellt sich in der Kinderund Jugendpsychiatrie die Arbeit im validen ökologischen Umfeld (Familie, Schule, Peergroup) immer wieder als sinnvoll, nachhaltig und effektiv dar. Multiple Studien haben dies nachgewiesen (Boege et al., 2014; Mattejat et al., 2001; Schmidt et al., 2006). Trotz der guten Evidenzlage gelang es aber bisher aufgrund der strikten Sektorengrenzen nicht, Hometreatment in Deutschland als Regelbe-

Tabelle 2. Erhaltene Leistung einer stationsäquivalenten Behandlung (STäB).

\begin{tabular}{|c|c|c|c|c|c|c|c|c|c|c|c|c|}
\hline \multirow[t]{2}{*}{ Störungsbilder } & \multirow[b]{2}{*}{$n$} & \multirow[b]{2}{*}{$\%$} & \multicolumn{2}{|c|}{ Kontakte gesamt } & \multicolumn{2}{|c|}{ Einzelgespräch } & \multicolumn{2}{|c|}{ Familiengespräch } & \multicolumn{2}{|c|}{ Fachtherapie } & \multicolumn{2}{|c|}{ Erlebnistherapie } \\
\hline & & & Kinder & Jugendl. & Kinder & Jugendl. & Kinder & Jugendl. & Kinder & Jugendl. & Kinder & Jugendl. \\
\hline Internalisierend & 42 & 72.4 & 36.0 & 41.2 & 19.2 & 23.5 & 7.1 & 6.5 & 4.9 & 5.5 & 4.9 & 5.9 \\
\hline Externalisierend & 16 & 27.6 & 35.9 & 47.5 & $15.9 *$ & $25.0 *$ & 6.9 & 8.2 & 6.3 & 5.8 & 5.8 & 8.5 \\
\hline Gesamt & 58 & 100 & 36.0 & 42.6 & $18.0 *$ & $23.8 *$ & 7.0 & 6.9 & 5.3 & 5.5 & 5.2 & 6.5 \\
\hline
\end{tabular}

Anmerkung. *signifikanter Unterschied mit $p<.05$

Tabelle 3. Kosten einer stationsäquivalenten Behandlung (StäB).

\begin{tabular}{|c|c|c|c|c|c|c|c|c|c|c|c|}
\hline \multirow[t]{2}{*}{ Störungsbilder } & \multirow[b]{2}{*}{$n$} & \multirow[b]{2}{*}{$\%$} & \multicolumn{3}{|c|}{ Kosten gesamt } & \multicolumn{3}{|c|}{ Fahrkosten } & \multicolumn{3}{|c|}{ Behandlungsleistungskosten } \\
\hline & & & Kinder & Jugendl. & $p$ & Kinder & Jugendl. & $p$ & Kinder & Jugendl. & $p$ \\
\hline Internalisierend & 42 & 72.4 & 8447.26 & 9094.52 & .586 & 879.69 & 1083.68 & .146 & 2225.46 & 2431.48 & .594 \\
\hline Externalisierend & 16 & 27.6 & 7813.08 & 10232.32 & .143 & 924.80 & 1370.96 & $.034 *$ & 1900.28 & 2602.18 & .117 \\
\hline Gesamt & 58 & 100 & 8228.58 & 9329.92 & .246 & 895.24 & 1143.12 & $.031 *$ & 2113.33 & 2466.80 & .238 \\
\hline
\end{tabular}

Anmerkung. OPS = Operationen- und Prozedurenschlüssel. * signifikanter Unterschied mit $p<.05$ 
handlungsangebot zu etablieren. Auch nach Inkrafttreten des PsychVVG 2017 hat sich Hometreatment in Form von StäB noch nicht flächendeckend etabliert. Fragen der Machbarkeit und Kostendeckung werden immer wieder aufgeworfen. Es sollte deswegen hier an den ersten konsekutiv behandelten 58 StäB-Fällen die Machbarkeit und Struktur einer StäB aufgezeigt werden. Der Familienkontext, in dem Kinder und Jugendliche leben, stellt dabei Idealbedingungen für die Indikation von StäB dar, da gerade bei Kindern der enge Einbezug des Umfeldes - im Sinne der Elternarbeit, Einbezug von Geschwistern und deren Rivalitäten, Einbezug von Schule und Schulproblemen wichtig für einen Behandlungserfolg ist, während bei Jugendlichen Themen wie Verantwortungsübernahme, Ablösung und Autonomiebestreben unter Berücksichtigung der elterlichen Kompetenzen eine Rolle spielen und am besten vor Ort gefördert werden sollten.

Die bisherige Erfahrung mit StäB zeigt, dass das Konzept von den Familien gut angenommen wird: $86.2 \%$ der stationsäquivalent behandelten Patientinnen und $\mathrm{Pa}-$ tienten und deren Eltern beendeten die Behandlung regulär, nur bei fünf Fällen musste eine Überleitung in eine vollstationäre Behandlung erfolgen, da die Intensität von StäB nicht ausreichend war (z. B. rascher Gewichtsverlust einer Patientin im Rahmen einer Anorexia nervosa mit Unterschreiten eines BMI von 13, aggressiv-verweigerndes Verhalten von einem Kind mit einer Störung des Sozialverhaltens und hoher hilfloser Erschöpfung der Eltern, Exazerbation einer psychotischen Erkrankung). Bei drei Patientinnen und Patienten kam es zum einvernehmlichen Beenden der Behandlung, da StäB nicht das richtige Angebot schien. 82\% der Familien wünschten sich noch eine niederschwelligere (1-mal/Woche) Nachbetreuung durch das StäB-Team, um die Behandlungseffekte zu festigen. Dies unterstreicht eine vorhandene Patientenzufriedenheit, betont allerdings auch, dass der Übertrag der erreichten StäB-Therapieziele in den Alltag auch bei aufsuchender Behandlung noch der weitergehenden zeitlich limitierten Nachbetreuung in Personalkontinuität bedarf. Die wenigsten Patientinnen und Patienten benötigten dabei mehr als zwei Quartale Nachbetreuung. Die Behandlungsdauer der intensiven StäB-Phase unterscheidet sich dabei nicht signifikant von der durchschnittlichen Behandlungsdauer einer stationären Regelbehandlung.

Der Erhalt des Alltags während der Behandlung wird in den bisherigen StäB-Fällen von den Familien, Kindern und Jugendlichen begrüßt und mit einer hohen Bereitschaft zur Mitarbeit sowie (terminlichen) Flexibilität beantwortet. Interessanterweise stellt sich in dem hier dargestellten Patientenkollektiv ein Fokus auf internalisierenden Störungen dar, obwohl in der Literatur eher externalisierende Störungen als besonders effektiv behan- delbar in einem intensivierten Hometreatment-Setting beschrieben wurden (Boege, Mayer et al., 2015; Henggeler et al., 1999; Rhiner, Graf, Dammann \& Fürstenau, 2011). Ein Vergleich mit den stationär aufgenommenen Patientinnen und Patienten in diesem Zeitraum ergab allerdings eine ähnliche Verteilung der Störungsbilder in StäB wie im stationären Bereich, sodass der geringere Anteil an Patientinnen und Patienten mit einer Störung des Sozialverhaltens nicht als spezifisch für StäB zu werten ist, sondern eher das Spektrum behandlungssuchender Familien, die sich mit dem Anliegen einer stationären Behandlung vorstellen, abbildet. Gegebenenfalls spielt bei dem Fokus auf internalisierenden Störungen auch die Eigenmotivation zur Behandlung eine Rolle, welche bei StäB eine Vorbedingung zur Aufnahme darstellt und bei Patientinnen und Patienten mit internalisierenden Störungen meist eher gegeben ist. Auch wird der Entlastungseffekt durch eine stationäre Aufnahme von Kindern und Jugendlichen mit externalisierenden Störungen von deren Eltern häufiger eingefordert als bei Kindern und Jugendlichen mit internalisierenden Störungen, was zu einer Vorauswahl der Patientinnen und Patienten, die zum Erstgespräch mit einem StäB-Mitarbeiter kamen, geführt haben mag. In StäB aufgenommene Patientinnen und Patienten mit externalisierenden Störungsbildern zeigten hingegen keine schwierigeren Behandlungsverläufe als diejenigen mit internalisierenden Problemen.

Auffällig war, dass Jugendliche in StäB signifikant mehr Einzelgespräche erhielten als Kinder, was einerseits der besseren altersbedingten kognitiven Fähigkeit sowie einem höheren Wunsch nach Abgrenzung gegenüber Eltern geschuldet sein mag, bei Jugendlichen im stationären Rahmen aber nicht automatisch so gegeben ist. Unabhängig von der Summe der Einzelgespräche spielten bei fast zwei Drittel aller Patientinnen und Patienten Kontaktanlässe in Bezug auf die Erziehung eine Rolle, sodass Elterngespräche und Elternanleitung durchgängig - unabhängig von der Altersstufe - mit im Fokus standen.

Inwiefern durch StäB mehr Kinder und Jugendliche mit psychischen Problemen erreicht werden können als mit den bisherigen Angebotsstrukturen, muss abgewartet werden. Dies deutet sich jedoch an, da die vollstationären Behandlungen in der Abteilung für Psychiatrie und Psychotherapie des Kindes- und Jugendalters am ZfP Südwürttemberg mit Beginn von StäB nicht zurückgegangen sind. Auch wurden Kinder und Jugendliche erreicht, die eine stationäre Aufnahme ablehnten, einer StäB aber zustimmten. Es wurden keine der vorgehaltenen vollstationären Betten reduziert, wobei anzumerken ist, dass die Bettenausstattung am ZfP Südwürttemberg pro minderjährigem Einwohner im baden-württembergi- 
schen Mittelfeld liegt. Baden-Württemberg wiederum liegt im untersten Quartil bundesweit.

StäB bedarf neben der Multiprofessionalität einer guten zeitgleichen Informationsweitergabe in Form einer angemessenen technischen Ausstattung und damit auch dezentralen Dokumentationsmöglichkeit, um der Komplexität der Behandlung in einem Team, das wenig persönliche Schnittstellen besitzt, an denen sich Teammitglieder face to face begegnen können, gerecht zu werden. Neben einer Ausstattung mit Laptops und Smartphones stellt die multiprofessionelle Fallbesprechung ein wichtiges Steuerungselement für die Koordination der therapiezielorientierten Behandlung der jeweiligen Fälle dar. Hier findet nicht nur Austausch, sondern auch die fachärztlich geleitete, supervidierte Behandlungsplanung statt. Deren Vorgabe in den StäB-Kriterien ist zu unterstreichen.

Trotz der Personalintensität, die für jede aufsuchende Behandlungsform vorgehalten werden muss, wurde bisher eine Kosteneffektivität von aufsuchender Behandlung in vorliegenden Studien nachgewiesen (Boege, Corpus et al., 2015; Kwok, Yuan \& Ougrin, 2016). Aktuelle erste Erfahrungen aus Pflegesatzverhandlungen zeigen, dass die Kostenträger nun auch bereit sind, ein solch intensives aufsuchendes Behandlungsangebot adäquat zu finanzieren. Die derzeit lokal verhandelten Entgeltwerte für StäBLeistungen bilden dabei die durchgeführten Behandlungselemente bisher kostendeckend ab. Interessanterweise stellte sich dar, dass Jugendliche mit externalisierenden Störungen höhere Kosten verursachen als Kinder mit externalisierenden Störungen. Dies ist möglicherweise dadurch erklärbar, dass bei Kindern mit externalisierenden Störungen weniger Einzelgespräche stattfanden, u.a. da die Aufmerksamkeitsspanne störungsspezifisch geringer war. Der Fokus lag auf Elterncoaching bzw. Elterntraining, welches, wie auch im stationären Rahmen, von Mitarbeitern des PED übernommen wurde. Bei Jugendlichen fanden ebenfalls Familiengespräche mit Elterncoaching statt, diese wurden aber in gleichem Ausmaß durch Einzelgespräche ergänzt.

\section{Limitationen}

Ziel dieser Studie war es, die Machbarkeit von StäB in den vorgegebenen gesetzlichen Rahmenbedingungen zu überprüfen. Dies erfolgte zunächst rein deskriptiv. Anhand der erhobenen Daten konnten deswegen noch keine Aussagen zu Effektivität von StäB und Stabilität der Behandlungsergebnisse von StäB im Verlauf erhoben werden. Ein randomisierter Vergleich mit einer stationären Behandlungsgruppe sowie eine genauere Kosten-Effektivitäts-Analyse sollte im Weiteren noch erfolgen.

\section{Fazit}

In dem hier dargestellten Patientenkollektiv stellt sich anhand der beobachteten Daten eine Machbarkeit von StäB in der vorgegebenen Bundesrahmenvereinbarung zwischen dem GKV-Spitzenverband, dem Verband der Privaten Krankenversicherung und der Deutschen Krankenhausgesellschaft (2017) dar. Das Konzept StäB wird von den infrage kommenden Familien, Kindern und Jugendlichen gut angenommen. Die täglichen Kontakte sind sowohl vonseiten des StäB-Teams als auch vonseiten der Familie abbildbar, bedürfen aber einer gegenseitigen Flexibilität. Weitere Studien sind nötig, um in Kosten-EffektivitätsAnalysen StäB mit stationären Behandlungen zu vergleichen sowie standardisiert Patientenzufriedenheit und Veränderungen im Funktionsniveau zu erheben.

\section{Literatur}

Barkmann, C. \& Schulte-Markwort, M. (2010). Prevalence of emotional and behavioural disorders in German children and adolescents: A meta-analysis. Journal of Epidemiology and Community Health, 66, 194-203.

Belfer, M.L. (2008). Child and adolescent mental disorders: The magnitude of the problem across the globe. Journal of Child Psychology and Psychiatry, 49, 226-236.

Boege, I., Corpus, N. \& Schepker, R. (2014). Behandelt zu Hause Gesund werden. Hometreatment in Verzahnung mit Klinikelementen, Chancen und Herausforderungen. Zeitschrift für Kinder- und Jugendpsychiatrie und Psychotherapie, 42, 27-37.

Boege, I., Corpus, N., Schepker, R., Kilian, R. \& Fegert, J.M. (2015). Cost-effectiveness of intensive homer treatment enhanced by inpatient treatment elements in child and adolescent psychiatry in Germany: A randomized trial. European Psychiatry, 30, 583-589.

Boege, I., Mayer, L., Muche, R., Corpus, N., Schepker, R. \& Fegert, J.M. (2015). Home treatment - insbesondere für expansive Jungen? Alters- und geschlechtsspezifische Wirksamkeit von Home treatment bei internalisierenden vs. externalisierenden Störungen. Zeitschrift für Kinder- und Jugendpsychiatrie und Psychotherapie, 43, 161-171.

Corpus, N., Schepker, R., Fegert, J.M. \& Boege, I. (2014). Eltern und Patienten als Subjekt der Behandlung - Erwartungen, Effizienz und Realitäten von intensiver, klinikverzahnter Behandlung zu Hause. Psychotherapeut, 59, 378-384.

Deutsche Krankenhausgesellschaft. (2017). Vereinbarung zur Stationsäquivalenten psychiatrischen Behandlung nach $\S 115 d$ Abs. 2 SGB V. Verfügbar unter https://www.dkgev.de/fileadmin/ default/Mediapool/2_Themen/2.3_Versorgung-Struktur/2.3.8._ Psychiatrie-Pyschosomatik/2.3.8.2._Stationsaequivalente_ psychiatrische_Behandlung/2017-08-01_Vereinbarung_Stati onsaequivalenteBehandlung.pdf

Gemeinsamer Bundesausschuss. (2018). Beschluss des Gemeinsamen Bundesausschusses zur Abnahme des Endberichts „Gutachten zur Weiterentwicklung der Bedarfsplanung i.S.d. $\S \S 99 \mathrm{ff}$. SGB V zur Sicherung der vertragsärztlichen Versorgung. Verfügbar unter https://www.g-ba.de/downloads/39-26 1-3493/2018-09-20_Endbericht-Gutachten-Weiterentwick klung-Bedarfsplanung.pdf 
Henggeler, S.W., Rowland, M.D., Randall, J., Ward, D.M., Pickel, S. G., Cunningham, P.B. et al. (1999). Home-based multisystemic therapy as an alternative to the hospitalization of youths in psychiatric crisis: Clinical outcomes. Journal of the American Academy of Child \& Adolescent Psychiatry, 38, 1331-1339.

Hölling, H., Erhard, M., Ravens-Sieberer, U. \& Schlack, R. (2007). Verhaltensauffälligkeiten bei Kindern und Jugendlichen: Erste Ergebnisse aus dem Kinder- und Jugendgesundheitssurvey (KiGGS). Bundesgesundheitsblatt Gesundheitsforschung Gesundheitsschutz, 50, 784-793.

Hölling, H., Schlack, R., Petermann, F., Ravens-Sieberer, U., Mauz, E. \& KiGGS Study Group (2014). Psychische Auffälligkeiten und psychosoziale Beeinträchtigungen bei Kindern und Jugendlichen im Alter von 3 bis 17 Jahren in Deutschland - Prävalenz und zeitliche Trends zu 2 Erhebungszeitpunkten (2003-2006 und 2009-2012). Bundesgesundheitsblatt, 57, 807-819.

Kwok, K.H.R., Yuan, S. N.V. \& Ougrin, D. (2016). Review: Alternatives to inpatient care for children and adolescents with mental health disorders. Child and Adolescent Mental Health, 21, 3-10.

Mattejat, F., Hirt, B. R., Wilken, J., Schmidt, M.H. \& Remschmidt, H. (2001). Efficacy of inpatient and home treatment in psychiatrically disturbed children and adolescents. Follow-up assessment of the results of a controlled treatment study. European Child and Adolescent Psychiatry, 10 (Suppl. 1), 71-79.

Mattejat, F., Simon, B., König, U., Quaschner, K., Barchewitz, C., Felbel, D. et al. (2003). Lebensqualität bei psychisch kranken Kindern und Jugendlichen. Zeitschrift für Kinder- und Jugendpsychiatrie und Psychotherapie, 31, 293-303.

Merikangas, K. R., He, J.P., Brody, D., Fisher, P.W., Bourdon, K. \& Koretz, D.S. (2010). Prevalence and treatment of mental disorders among US children in the 2001-2004 NHANES. Pediatrics, 125, 75-81.

Ravens-Sieberer, U., Schulte-Markwort, M., Bettge, S. \& Barkmann, C (2002). Risiken und Ressourcen für die psychische Gesundheit von Kindern und Jugendlichen. Gesundheitswesen, 64 (Suppl. 1), 88-94.

Ravens-Sieberer, U., Wille, N. \& Erhard, M. (2008). Prevalence of mental health problems among children and adolescents in Germany: Results of the BELLA study within the National Health Interview and Examination Survey. European Child and Adolescent Psychiatry, 17 (Suppl. 1), 22-33.

Remschmidt, H., Schmidt, M. H., Mattejat, F., Eisert, H.G. \& Eisert, M. (1988). Therapieevaluation in der Kinder- und Jugendpsychiatrie: Stationäre Behandlung, tagesklinische Behandlung und Home Treatment im Vergleich, Zeitschrift für Kinder- und Jugendpsychiatrie und Psychotherapie, 16, 124-134.

Rhiner, B., Graf, T., Dammann, G. \& Fürstenau, U. (2011). Multisystemische Therapie (MST) für Jugendliche mit schweren Verhaltensstörungen. Implementierung in der deutschsprachigen Schweiz und erste Ergebnisse. Zeitschrift für Kinder- und Jugendpsychiatrie und Psychotherapie, 39, 33-39.
Schmidt, M.H., Lay, B., Göpel, C., Naab, S. \& Blanz, B. (2006). Hometreatment for children and adolescents with psychiatric disorders. European Child and Adolescent Psychiatry, 15, 265-76.

Signorini, G., Singh, S.P., Boricevic-Marsanic, V., Dieleman, G., Dodig-Curcovic, K., Franic, T. et al. (2017). Architecture and functioning of child and adolescent mental health services: A 28-country survey in Europe. Lancet Psychiatry, 4, 715-724.

Statista. (2017). Gesamtversorgungsgrade der Kinder- und Jugendpsychiater in Deutschland nach Bundesländern im Jahr 2016. Verfügbar unter https://de.statista.com/statistik/daten/ studie/601998/umfrage/gesamtversorgungsgrade-der-kinderund-jugendpsychiater-nach-bundeslaendern/

Statistisches Bundesamt. (2018). Gesundheit. Grunddaten der Krankenhäuser - Fachserie 12 Reihe 6.1.1 - 2017. Verfügbar unter https://www.destatis.de/DE/Themen/Gesellschaft-Umwelt/Gesundheit/Krankenhaeuser/Publikationen/DownloadsKrankenhaeuser/grunddaten-krankenhaeuser-21206111770 04.pdf?__blob=publicationFile

Warnke, A. \& Lehmkuhl, G. (2011). Kinder- und Jugendpsychiatrie und Psychotherapie in der Bundesrepublik Deutschland: Die Versorgung von psychisch kranken Kindern, Jugendlichen und ihrer Familien. Stuttgart: Schatthauer.

Wille, N., Bettge, S., Wittchen, H.U. \& Ravens-Sieberer, U. (2008). How impaired are children and adolescents by mental health problems? Results of the BELLA study. European Child and Adolescent Psychiatry, 17 (Suppl. 1), 42-51.

\section{Historie}

Manuskript eingereicht: 10.10.2019

Nach Revision angenommen: 04.01.2020

Onlineveröffentlichung:25.02.2020

\section{Interessenkonflikt}

Die Autoren haben keinen Interessenkonflikt.

\section{Isabel Boege, PD Dr.}

ZfP Südwürttemberg

Akademisches Lehrkrankenhaus der Universität Ulm

Weingartshoferstr. 2

88214 Ravensburg

Deutschland

isabel.boege@zfp-zentrum.de

isabel.boege@t-online.de 